\title{
Numerical Triaxial Apparatus and Application
}

\author{
Xiaoliang Wang ${ }^{1, a}$, Jiachun $\mathrm{Li}^{2, b}$ \\ ${ }^{1,2}$ Insititute of Mechanics, Chinese Academy of Sciences, No.15 Beisihuanxi Road, Beijing, China \\ âwangxiaoliang@imech.ac.cn
}

Keywords: DEM, Granular Materials, Yade, triaxial

\begin{abstract}
A numerical triaxial apparatus based on discrete element method is developed on the platform of Yade using Python script. A DEM model with rolling resistance contact considered is proposed for dense granular materials, which is then applied in triaxial compression test of Chende sand. Stress-strain response and volume-axial strain response of the DEM model agree well with that of experiments, with a good prediction of dilatancy angle. Degradation of granular materials duo to particle erosion is also investigated using triaxial compression tests. It is indicated that peak friction angle decreases with the remove of particles if strong force network of granular materials is destroyed.
\end{abstract}

\section{Introduction}

Granular materials, like sand and clay, made of an assemblage of independent elements (particles, block), are ubiquitous in nature. They are always used as foundation to support load in civil engineering or construction materials, benefit to mankind. On the other hand, they are the media of natural disasters, in landslides, avalanche and debris flow, in which a massive of granular materials fail, bringing catastrophic loss to people, especially after a big rain ${ }^{[1]}$. As materials of a slope, made of sand, gravel, clay, rock etc., granular materials behave sensitive to water, especially for the weak band of a slope. Sand and gravel are considered as cohesionless materials, whose mechanical behavior depends on particle size, particle shape, particle mineral and relative density etc., usually not as sensitive as that of clay to water. Constitutive relationships and strength are needed in geotechnical engineering prediction and design. Direct shear test is often used to obtain strength parameters. Another equipment is triaxial compression apparatus, which is used to investigate behavior of granular materials. There are difficulties in direct shear test and triaxial compression test, like how to prepare granular materials sample, especially intact sample, a waste of human resource and money, etc. The last 30 years of progress of discrete element method (DEM) since $1979^{[2]}$ has provided another, at least a complementary tool in investigating behavior of granular materials. With the development of hardware of computer and software engineering, now a number of open sources based on DEM, like Yade ${ }^{[3]}$, ESyS-particle ${ }^{[4]}$ and LIGGGHTS ${ }^{[5]}$ etc., have provided researchers platforms in investigating behavior of granular materials. In this paper, a numerical triaxial apparatus is developed on the platform of Yade using Python script, which is then applied to investigate dilatancy of dense granular materials and degradation of granular materials duo to erosion.

\section{Numerical Triaxial Apparatus}

Mechanics of Granular Materials. Granular materials are composed of an assemblage of solid particles, with void occupied by water or air. From point view of mechanics, behavior of granular materials is an average of motions and geometries of all particles of a representative element volume (REV). In an assemblage, one particle interacts with its surrounding particles through 
normal compression, shear friction, moment resistance, physical-chemical interactions ${ }^{[6]}$. If water effect plays role, surface force and hydrodynamic drag should also be considered. Besides, motions of each particle, including translation and rotation, are governed by Newton's second law and Euler's equation. All the mechanical behaviors, for example, stress-strain behavior and shear dilatancy relation, are obtained through average over all particles of the REV.

Discrete Element Method. As stated previously, mechanical behavior of granular material is complex duo to its particle structures. With the power of computer science and numerical algorithm, including searching algorithm and finite difference algorithm, it is possible to solve equations of dynamics of thousands of particles in a PC or a small workstation numerically. Discrete element method, considers granular materials as an assemblage of particles, and solves motion of every particle numerically. Shapes of particles in reality are complex, leading to complex interaction between which, So simplification of geometry and interaction is necessary in DEM. The simplest geometries are circles in 2D and spheres in 3D, respectively, which is applied in most of the DEM codes as fundamental particle. Interaction between two particles is simplified by a spring and a dashpot, or hertz interaction. Another advantage of circle or spherical particle is that searching interaction time is a bit less than that of complex shapes. A simple method to model non-spherical particle is to glue a series of particles together as a super particle. Besides, there is also polyhedron, elliptical particle, but searching interaction time for them is very expensive.

Yade Platform and Python Interface. Yade ${ }^{[3]}$ is an open-source platform now available for all researchers with a python script interface. Yade uses sphere as its fundamental sphere, and clumped sphere to model non-spherical particles, with some stable algorithms to generate granular sample. Linear interaction, Hertz interaction and cohesive interaction between particles are provided in Yade. In addition, water effects for unsaturated granular materials could be considered in Yade now $^{[7]}$. Servo algorithm is also provided in Yade for wall plates to keep a normal pressure on which. Besides, there are two functions for triaxial compressions. Users can use different boundaries, including periodical boundary, fixed wall plate, and wall plate with a prescribed pressure. There are also some post-process functions available for users to plot force chains, Y-X plots, etc. All of the functions can be operated in a Python script. In the platform of Yade, using Python interface, we have designed a numerical triaxial apparatus, with some special utilities. One can define his particle assemblage using his particle size distribution (psd). Elastic interaction, friction interaction, cohesive interaction, rolling resistance, and capillary interaction can be added in between particles. Conventional triaxial compression tests and suction controlled triaxial compression tests can be operated. Stress and strain can be easily obtained and exported to files, which can be then analyzed by matlab, Python and Paraview etc. Another subroutine is designed to calculate fabric tensor of granular materials.

\section{Application in Granular Materials}

Dilatancy of Granular Media. Dilatancy is a phenomenon of dense and medium dense sand. When shearing, particles need to roll over other particles supported them, resulting volume expansion. In DEM, dense sand is modeled as an assemblage of spherical particles. If only normal and shear spring with a friction threshold considered, the dilatancy angle is a bit larger (often $30^{\circ}$ ) than that of true dense sand $\left(10^{0}\right.$ for Chende sand $\left.^{[8]}\right)$. Spherical particles roll easier than non-spherical particles, so spherical particle model underestimates friction angle and overestimates the dilatancy angle. A compromise way used here is to add a rolling resistance to the interaction to model the moment transmitted during rolling of the two contacting particles ${ }^{[9]}$. A DEM model considered rolling resistance, is applied to model Chende sand, which is a medium dense to dense 
sand. Calibration procedure is omitted here duo to the page limitation. The sample is generated by radius expansion method ${ }^{[10]}$, which is a stable packing algorithm. Then triaxial compression is taken over the sample until the axial strain is $10 \%$. Stress-strain curves and volume-axial strain responses of confining stresses $100 \mathrm{kPa}$ and $300 \mathrm{kPa}$ are shown in Fig.1 and Fig.2, respectively, together with results of Li's experiment ${ }^{[8]}$. Results of our DEM model agree well with that of Li's experiment. Friction angle is $36.2^{\circ}$ of the DEM model, compared to $35.3^{\circ}$ of the experiment. Dilatancy angles of DEM model are $8.6^{\circ}$ and $11.7^{\circ}$, of confining stress $100 \mathrm{k}$ and $300 \mathrm{kPa}$, and those of experiments are $10.1^{0}$ and $12.8^{0}$.

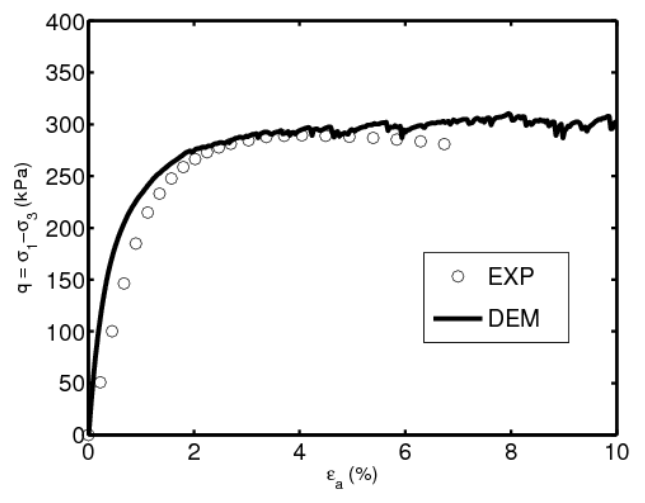

(a)

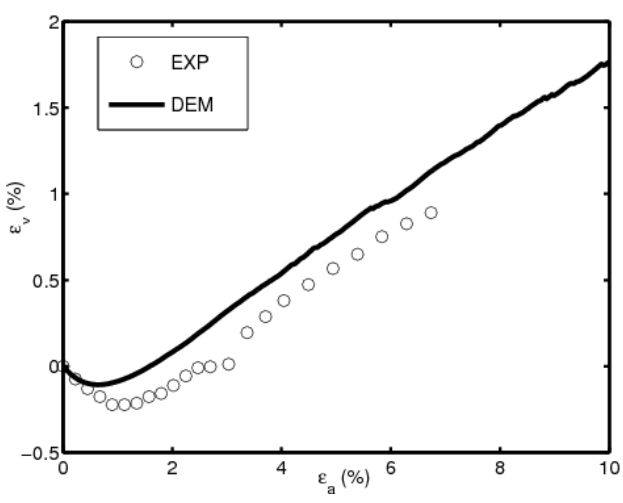

(b)

Fig.1 Results of triaxial compression test of Chende sand. Stress-strain behavior (a), volume-axial strain response (b). Confining pressure is $100 \mathrm{kPa}$.

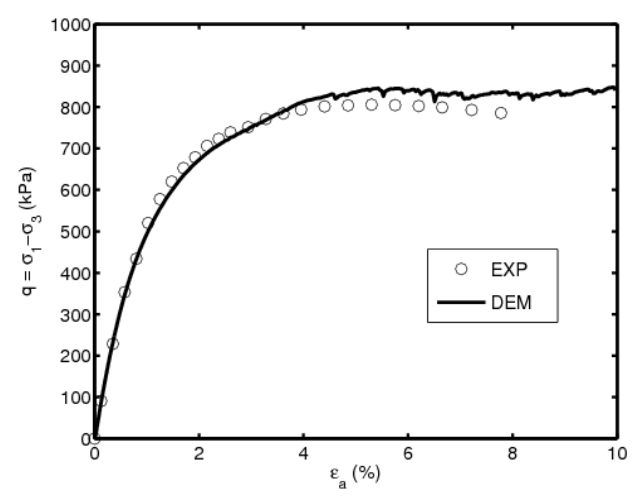

(a)

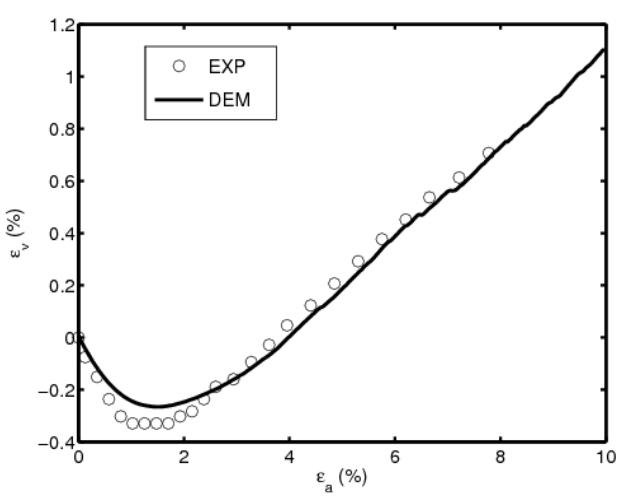

(b)

Fig.2 Results of triaxial compression test of Chende sand. Stress-strain behavior (a), volume-axial strain response (b). Confining pressure is $300 \mathrm{kPa}$.

Degradation of Granular Media Due to Erosion. In some cases of geotechnical engineering, granular materials suffer erosion under the interaction of water. In piping disaster of a dam, under a limit hydrodynamic gradient, fine particles are flooded away by flow, causing a degradation of granular materials, which often leads to dam failure. Experiment of how the mechanical behavior degrades with the erosion of particles is almost impossible in the conventional triaxial compression tests, but very easy in our numerical triaxial apparatus, because particle erosion is easy in numerical world. In this study, a dense sample of granular materials with a good psd defined by normalized incomplete beta function ${ }^{[11]}$ is chosen. After isotropic compression, a specified percentage of mass, radius of which is taken from the smallest, is removed at a specified vertical stress, then triaxial compression is kept on to an axial strain of $20 \%$. It is found that the stress state where particles are 
removed does not influence the stress-strain response. Fig.3 shows the stress-strain and volume-axial strain responses, mass eroded of which is $1 \%, 3 \%, 5 \%$ and $8 \%$, respectively. It is indicated that peak friction angle decreases with the particle erosion. After the small particles are removed, granular packing becomes loose, so the dilatancy also becomes smaller and smaller, which is the key to the peak friction degradation. Macroscopic stress is an average of the inter-particle contact forces, while inter-particle forces are decomposed into a strong and a weak force, the former larger than the average force, while the latter one smaller. It is indicated in Fig. 4 that the degradation of granular materials is mainly due to degradation of strong force networks. In piping disasters, small particles play a less role in supporting the dam, but to stable the strong force networks, so small particles are easy to be removed by water flow. If water flow begins to destroy the particles structure made of strong force networks, granular materials begin to degrade.

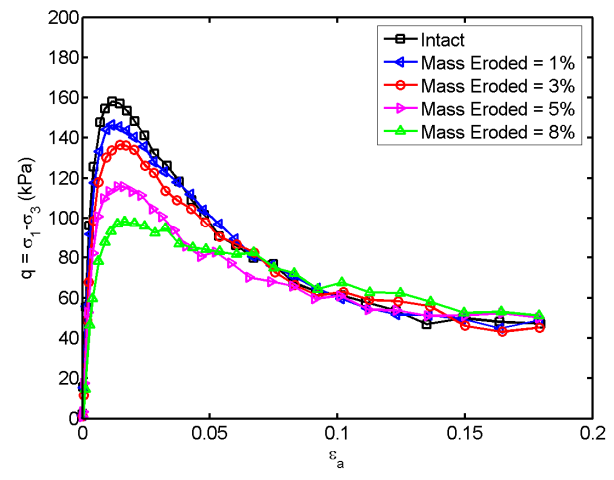

(a)

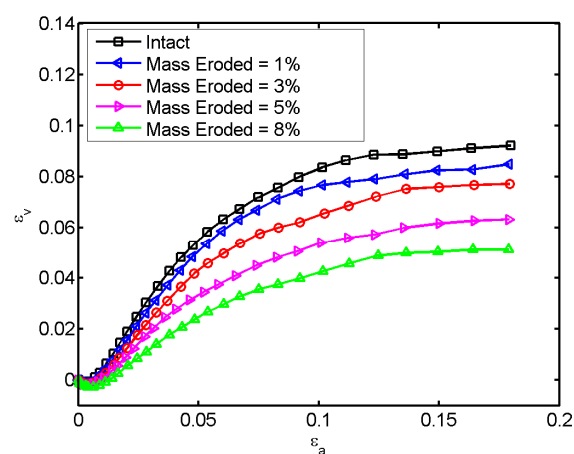

(b)

Fig.3 Responses of triaxial compression tests duo to particle erosion. (a) stress-strain response,

(b) volume-axial strain response.

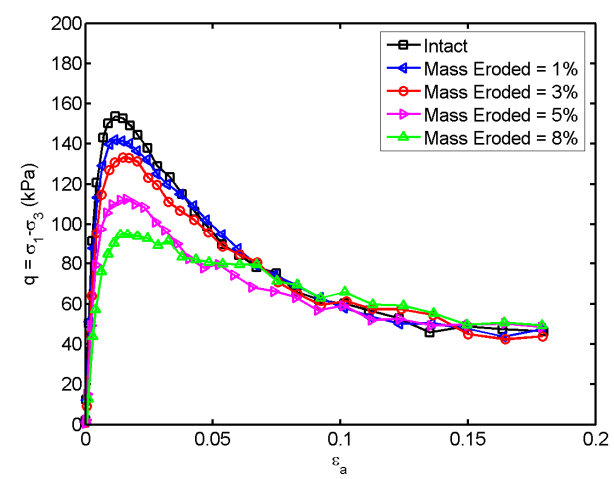

(a)

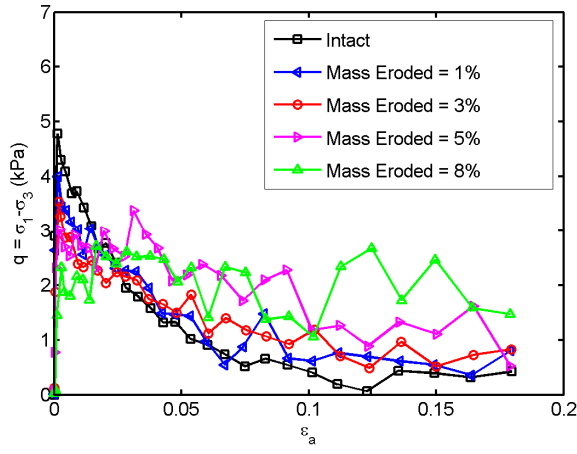

(b)

Fig.4 Contributions of strong inter-particle force (a) and weak inter-particle force (b).

\section{Conclusion}

A numerical triaxial compression apparatus with various utilities is developed on the platform of Yade using Python script, with two applications of behavior of granular materials. The DEM model with rolling resistance is a suitable way to model dense to medium dense granular materials, especially to predict the dilatancy angle. Granular materials will suffer degradation if particles are removed until strong force networks are destroyed. 


\section{Acknowledgements}

We appreciate the foundation provided by National Natural Science Foundation of China in project "Mechanism of failure of soil and rock under interaction of water" (Grant No.10932012 ). The first author wants to acknowledge Chareyre etc. for the discussion on mechanics of granular materials on YADE launchpad.

\section{References}

[1] S. Lignon, F. Laouafa, F. Prunier, H. D. V. Khoa and F. Darve: Geotechnique 59 (6), 513-524 (2009).

[2] P. A. Cundall and O. D. L. Strack, Geotechnique 29 (1): 47-65 (1979).

[3] J. Kozicki and F. V. Donze: Comput. Method Appl. M. 197 (49-50), 4429-4443 (2008).

[4] Information on http://espace.library.uq.edu.au/view/UQ:187819.

[5] C. Kloss and C. Goniva: Supplemental Proceedings, 781-788 (2011).

[6] H. Zhu, Z. Zhou, R. Yang and A. Yu: Chem. Eng. Sci. 62 (13), 3378-3396 (2007).

[7] L. Scholtes, P. Y. Hicher, F. Nicot, B. Chareyre and F. Darve: Int. J. Numer. Meth. Eng. 33 (10), 1289-1313 (2009).

[8] G. X. Li, 3D Constitutive Relationships for soils and experiment validation, Tsing Hua University, 1985 (in Chinese).

[9] N. Belheine, J. P. Plasslard, F. V. Donze, F. Darve and A. Seridi: Comput. Geotech. 36 (1-2), 320-331 (2009).

[10] F. Radjai and F. Dubois, Discrete-Element Model of Granular Materials. (Wiley, New York 2011).

[11] C. Voivret, F. Radjai, J. Y. Delenne and M. El Youssoufi: Physical Review E 76 (2), 021301 (2007). 